\title{
Is this the end of cytotoxic chemotherapy in Merkel cell carcinoma?
}

This article was published in the following Dove Press journal:

OncoTargets and Therapy

28 September 2017

Number of times this article has been viewed

\section{Guilherme Rabinowits}

Department of Medical Oncology, Center for Head and Neck Oncology, Dana-Farber Cancer Institute, Boston, MA, USA
Correspondence: Guilherme Rabinowits Center for Head and Neck Oncology, Dana-Farber Cancer Institute, 450 Brookline Avenue, Boston, MA 02215, USA

Tel + I 6176323090

Fax +I 6176324448 Email guilherme_rabinowits@dfci. harvard.edu

\begin{abstract}
Although cytotoxic chemotherapy has been used often in the management of Merkel cell carcinoma (MCC), its benefit remains uncertain. Despite being considered a chemosensitive disease, the duration of response is generally short, and the survival benefit is unclear. With the recent FDA approval of the anti-programmed cell death ligand 1 (PD-L1) antibody avelumab for patients with advanced MCC and the limited and controversial data on chemotherapy, it is important to put in perspective whether conventional chemotherapy should remain an option for these patients. Here, we review the evidence and controversies around chemotherapy in MCC as well as two recent studies on immunotherapy that changed the treatment paradigm for this disease.
\end{abstract}

Keywords: Merkel cell carcinoma, chemotherapy, immunotherapy, review, future

\section{Introduction}

Merkel cell carcinoma (MCC) is a rare and aggressive neuroendocrine cutaneous malignancy managed primarily with surgery and radiation therapy, despite its high metastatic potential. ${ }^{1}$

Approximately half of the patients who present with locally advanced disease die of recurrence or metastasis. ${ }^{2}$ The highest risk of recurrence is within the first 2 years following treatment, with most recurrences happening outside the radiation treatment fields, causing some to consider MCC a systemic disease regardless of its stage at presentation. ${ }^{1,3} \mathrm{MCC}$ is known to be a chemosensitive disease, with reported response rates as high as $60 \%-75 \% .{ }^{4}$ Commonly used agents include platinum, etoposide, cyclophosphamide, vincristine, doxorubicin, and topotecan, alone or in combination. ${ }^{4,5}$ Chemotherapy is generally used for the treatment of local and/or regional recurrence ${ }^{4}$ or distant disease, ${ }^{5}$ although it is often given in the adjuvant setting - despite unclear survival benefit. ${ }^{3,6}$ A single-institution, retrospective study of 62 patients with metastatic MCC who received first- and second-line chemotherapy reported a response rate of 55\% and 23\% and a median progression-free survival of 94 and 61 days among patients, respectively, and a median overall survival of only 9.5 months. ${ }^{5}$ The relatively short duration of response seen in the metastatic setting, significant treatment-related toxicities, and concerns regarding immunosuppression cause many to consider chemotherapy as the "last resort" for these patients and underlines the urgent need for enhanced treatment options for this disease. ${ }^{4,5}$

\section{Main evidence and controversies of chemotherapy in the adjuvant treatment of MCC}

Interpretation of studies on the use of adjuvant chemotherapy in MCC is challenging due to the lack of prospective randomized trials, limited experience, different 
chemotherapy regimens used, heterogeneous patient population, small sample size, and short follow-up.

To date, only two prospective trials have evaluated the role of chemotherapy in the adjuvant setting. The first study was a Phase II trial of synchronous chemoradiation therapy in high-risk MCC patients (TROG 96:07) that was conducted by the Trans-Tasman Radiation Oncology Group. ${ }^{7}$ High risk was defined as primary tumors $>1 \mathrm{~cm}$ in size, recurrence following initial surgery, gross residual disease after surgery, or occult primary with nodal involvement. Wide surgical clearance of the primary site was not required nor recommended, and it was not a prerequisite to have nodal disease resected or positive margins re-excised. Radiotherapy was given for 5 weeks, with combination chemotherapy consisting of carboplatin and etoposide administered during weeks 1 and 4 of radiotherapy and at weeks 7 and 10 . Fifty-three patients were enrolled: $12(23 \%)$ patients presented with recurrence after prior therapy; 13 (25\%) had unknown primary disease; and $33(62 \%)$ had nodal disease. Fifteen (28\%) patients received chemoradiotherapy as definitive therapy. Fortysix $(87 \%)$ patients received all four doses of chemotherapy. Grade 3-4 skin reactions and febrile neutropenia occurred in $64 \%$ and $35 \%$ of patients, respectively. No treatment-related deaths occurred. The 3-year overall survival was 76\% (83\% for occult primary and $74 \%$ for known primary disease); relapse-free survival was $65 \%$; locoregional control was $75 \%$ (91\% for occult primary and 70\% for known primary disease); and distant control was $76 \%$ (91\% for occult primary and $56 \%$ for known primary disease). Locoregional failure at the primary site, in-transit areas, or draining nodes occurred in $9(17 \%)$ of the 53 patients; 7 in the absence of distant disease. Patients with occult primary disease were considered high-risk patients and eligible for this trial, and had a better prognosis in comparison with those with known primary disease. The excellent outcome seen in the unknown primary MCC group could be attributed, at least in part, to the chemotherapy, but the lower distant control seen in the known primary disease group argues against a chemotherapy effect. Moreover, other studies have demonstrated a better outcome for patients with unknown primary MCC, regardless of whether chemotherapy was administered. ${ }^{8,9}$

In an attempt to better clarify the effect of chemotherapy in the adjuvant setting, Poulsen et al retrospectively compared the data of patients with known primary MCC from the TROG 96:07 study with the results of patients with known primary MCC from the Queensland Radium Institute database who would have been otherwise eligible to participate in the TROG 96:07 study, but were treated without chemotherapy. The patients selected from the database had no evidence of disease beyond regional nodes and had high risk of recurrence (primary size $>1 \mathrm{~cm}$, involved nodes, recurrence after initial surgery, or gross residual disease after surgery). ${ }^{10}$ Of a total of 102 patients, 40 were treated with chemotherapy as part of the TROG 96:07, and 62 were treated without chemotherapy in the historic control group. Although cancer-related and cancer-unrelated deaths - as well as recurrences in locoregional and distant sites - were lower in the chemotherapy group, no statistically significant difference was detected in disease-specific survival, local and distant control, and overall survival (hazard ratio [HR] $0.64 ; 95 \%$ confidence interval [CI] $0.34-1.2$; $p=0.16$ ) in multivariate analysis between the groups. The authors suggested the small number of patients, in addition to the large imbalance in measured and possibly unmeasured covariates, as possible explanations for this lack of difference between the groups.

A second prospective study was initiated in order to decrease the risk of neutropenic fever seen in the TROG 96:07 trial. This Australian study was launched utilizing weekly carboplatin concurrently with radiotherapy, followed by three cycles of adjuvant carboplatin and etoposide combination for local or locally advanced, high-risk MCC. ${ }^{11}$ High risk was defined as previously described. Eighteen patients (14 with known primary) were enrolled. No patient developed a relapse in the primary site, but in-transit recurrences developed in one patient, nodal metastasis in four (only one within the radiation field), and distant relapse in three patients. No treatment-related deaths occurred. Grade 3 skin and neutrophil toxicities occurred in 23\% and $25 \%$ of patients, respectively, in contrast to the $65 \%$ and $57 \%$ seen in the TROG 96:07, respectively. In addition, weekly carboplatin did not appear to have a detrimental effect on tumor control, suggesting it may be a better regimen if concurrent chemoradiation therapy is planned.

In another study, Allen et al reported a single-institution retrospective review of 251 patients managed between 1970 and 2002. ${ }^{3}$ One hundred and seventy-seven (70\%) patients presented with clinically negative regional nodes, 60 (24\%) with clinically suspicious regional lymphadenopathy, 14 (6\%) with distant metastatic disease, and 31 (12\%) with unknown primary tumor. Adjuvant radiation therapy was administered to $41(17 \%)$ patients, and adjuvant chemotherapy to $28(12 \%)$ of the 237 patients who presented with local or regional disease, the majority ( $30 \%$ vs $2 \%$ ) with node-positive disease. The 5-year relapse-free survival for these 237 patients was $48 \%$. The most common site of recurrence for all patients 
was the draining nodal basin. On the group of patients with node-positive disease (23 patients received chemotherapy and 53 patients did not), the use of adjuvant chemotherapy was not associated with disease-specific survival.

More recently, a retrospective analysis of 6,908 MCC cases from the National Cancer Database (NCDB) has been conducted to assess whether adjuvant therapy was associated with difference in survival. ${ }^{6}$ Age, sex, primary MCC site and tumor size, comorbidity, margin and lymph node evaluation status, extent of lymph node resection, and the number of positive nodes were included in analyses. Information on the type of surgery (wide excision or other), sites of radiation therapy (primary site and/or nodal), chemotherapy dose and regimen, and disease-specific mortality were not available. Increasing age, male sex, presence of comorbidities, positive margin status, and increasing tumor burden (reflected by the primary tumor size in stages I and II and the number of positive nodes in stage III) were associated with poorer survival across all stages. Surgery with adjuvant radiation therapy was associated with statistically significant better overall survival than surgery alone on multivariate analysis for patients with stage I $(\mathrm{n}=3,369)$ and stage II $(\mathrm{n}=1,474)$ disease $(p<0.001)$. In contrast, neither adjuvant radiation therapy nor chemotherapy was associated with statistically significant improvement or worsening survival in patients with stage III $(n=2,065)$ disease. Although the lack of benefit with adjuvant chemotherapy was consistent with those reported in prior studies, the lack of benefit with radiation therapy for stage III disease was surprising, given the improvement in locoregional control and survival seen in other studies. ${ }^{12-14}$ The authors hypothesized that survival in stage III was mostly driven by the presence of subclinical distant metastases that are often present in these patients. If that is true, one would expect similar results in those with stage III, unknown primary MCC; however, the better prognosis seen in these patients argues against that. Moreover, the lack of information on disease-specific mortality and other confounders, such as immunosuppression, that may impact the prognosis in these patients as well as details with regard to the administration of various treatment modalities further complicates the interpretation of these findings. A randomized prospective trial is required to better answer these questions.

To date, only one study demonstrated a survival benefit with the use of chemotherapy in a subset of MCC patients. Chen et al evaluated the role of adjuvant therapy in 4,815 patients with head and neck MCC from the NCDB. ${ }^{15}$ Of these, 2,330 patients underwent adjuvant chemoradiation therapy, 330 patients underwent adjuvant radiation therapy alone, 97 received adjuvant chemotherapy only, and 1,995 were treated with surgery alone. The 5 -year overall survival was $41 \%$. Postoperative chemoradiation therapy (HR 0.62; 95\% CI 0.47-0.81) and radiation therapy (HR 0.8; 95\% CI $0.7-0.92$ ) provided a survival benefit over surgery alone. Adjuvant chemoradiation therapy was associated with improved overall survival over adjuvant radiation therapy in patients with positive margins (HR 0.48 ; 95\% CI $0.25-0.93$ ), tumor size of at least $3 \mathrm{~cm}$ (HR $0.52 ; 95 \%$ CI $0.3-0.9$ ), and male sex (HR $0.69 ; 95 \%$ CI $0.5-0.94$ ), suggesting that a subgroup of patients may truly benefit from the addition of chemotherapy to the treatment regimen.

The National Comprehensive Cancer Network (NCCN) Clinical Practice Guidelines for MCC suggest that the use of adjuvant chemotherapy should be considered on a caseby-case basis, in particular for those with local macroscopic or regional disease, although it is unclear whether a survival benefit can be achieved based on currently available data. ${ }^{16}$

\section{Immunotherapy in MCC}

Immunosuppression is a well-known risk factor for MCC and has been correlated with worse prognosis for these patients. ${ }^{17}$ Other risk factors include Caucasian ethnicity, advanced age, ultraviolet exposure, and the Merkel cell polyomavirus (MCPyV) ${ }^{18,19} \mathrm{MCPyV}-$ negative $\mathrm{MCC}$ has a characteristic ultraviolet signature and a distinct mutational profile - with over 30-fold more somatic mutations, many more copy number alterations, and interchromosomal translocations - when compared to MCPyV-positive tumors. ${ }^{20}$ Although weak MCPyV T-cell responses may explain the development of the cancer, programmed cell death ligand 1 (PD-L1) expression has been shown in MCC cells and adjacent immune cell infiltrates. ${ }^{21}$ PD-L1 is a key therapeutic target in the reactivation of the immune response and a promising target in cancer therapy. Additionally, tumor-infiltrating $\mathrm{CD}^{+}$and $\mathrm{CD}^{+}{ }^{+} \mathrm{T}$ cells specific to $\mathrm{MCPyV}$ oncoproteins are enriched in some MCC in association with enhanced expression of both PD-L1 and its receptor - the programmed cell death 1 (PD-1) receptor. The expression patterns of immunerelated inhibitory markers in MCPyV-positive tumors and the high mutational load seen in MCPyV-negative tumors provide a rationale for treatment with immune checkpoint inhibitors in MCC.

Indeed, immunotherapeutic activity has been recently demonstrated in both chemotherapy-naïve and chemotherapyrefractory MCC patients, with a favorable toxicity profile, duration of response, and progression-free survival when compared to results seen with cytotoxic chemotherapy. ${ }^{22,23}$ 
Table I Patient and tumor characteristics of avelumab and pembrolizumab studies

\begin{tabular}{lll}
\hline Studies characteristics & Avelumab $^{23}$ & Pembrolizumab $^{22}$ \\
\hline Phase & II & II \\
\hline Mechanism of action & lgGI monoclonal antibody blocking & IgG4 monoclonal antibody blocking \\
& PDI/PD-LI interaction; ADCC & PDI/PD-LI and PDI/PD-L2 interactions \\
Target & PD-LI & PDI \\
Number of patients & 88 & 26 \\
Median age (years) & 72.5 & 68 \\
Disease stage & IV & IIIB/IV \\
Prior lines of therapy & $\geq I$ & 0 \\
MCPyV-positive, $n(\%)$ & $46(52)$ & I7 (65) \\
PD-LI positive, $n(\%)$ & $58(66)$ & I4 (56) \\
\hline
\end{tabular}

Abbreviations: IgG, immunoglobulin G; ADCC, antibody-dependent cell-mediated cytotoxicity; PDI, programmed cell death I; PD-LI, programmed cell death ligand I; PD-L2, programmed cell death ligand 2; MCPyV, Merkel cell polyomavirus.

A landmark Phase II study evaluating the anti-PD1 antibody pembrolizumab in 26 patients with metastatic MCC demonstrated a response rate of 56\% (62\% in virus-positive and $44 \%$ in virus-negative disease) and a median progression-free survival of 9 months. ${ }^{22}$ Tumor regression was seen regardless of the level of PD-L1 expression. Although treatment-related adverse events were reported in $77 \%$ of the patients, the majority were grade 1 and 2 events. Fatigue and laboratory abnormalities were the most commonly occurring adverse events. Results of this trial led to the addition of pembrolizumab in the most recent update of the NCCN Clinical Practice Guidelines as a treatment option for MCC patients with advanced disease. ${ }^{16}$

On May 23, 2017, the anti-PD-L1 antibody avelumab received FDA approval for patients with advanced MCC after a seminal study demonstrated a response rate of $31.8 \%$ in 88 patients with stage IV MCC that had failed one or more lines of systemic therapy. ${ }^{23}$ The median follow-up of these patients was 10.4 months, and $82 \%$ of the responders continued to respond at the time of analyses. Treatment-related adverse events were reported in $70 \%$ of the patients, the majority being of grades 1 and 2, with the most common being fatigue and infusion-related reactions. Results of this trial have been recently updated indicating that, at a median follow-up of 16.4 months, the response rate was $33 \%$, including 10 complete and 19 partial responses, with the median duration of response not yet reached..$^{24}$ Interestingly, response rates to avelumab appear to be higher for those with no prior lines of systemic therapy in contrast to those with one line or more than one line of prior systemic therapy ( $56.3 \%$ vs $40.4 \%$ vs $19.4 \%$, respectively). ${ }^{23,25}$

Neither the avelumab nor the pembrolizumab studies demonstrated a statistically significant difference in treatment responses based on MCPyV status or PD-L1 expression. ${ }^{22,23}$ Although activity and toxicity of avelumab and pembrolizumab appear similar, that can only be confirmed on a randomized prospective trial. Moreover, it is unclear whether disease resistance to one agent can be overcome by the other. Regardless, only avelumab is currently approved for MCC and considered the treatment of choice for those with advanced disease without contraindications for immunotherapy. Table 1 summarizes the differences between patient and tumor characteristics of the avelumab and pembrolizumab MCC studies.

Despite the excitement with the immune checkpoint blockade, it is important to remember that many patients will not benefit from, or are not candidates for, immunotherapy, leaving them without optimal systemic therapy options.

\section{Conclusion}

Despite the exciting results seen with immunotherapy and the recent FDA approval of avelumab in advanced MCC, it is not the end of cytotoxic chemotherapy in MCC. Although it remains a palliative option for those with metastatic disease, immunotherapy will likely leave chemotherapy as an option for those who are not candidates for or progressed after immunotherapy, or those who are not eligible for a clinical trial. Given the high response rates seen with chemotherapy, it is possible that responses may improve when combined with targeted agents or immune checkpoint inhibitors, an area of active research interest in oncology. It is important to emphasize that chemotherapy does result in disease shrinkage or control in a number of patients. Although temporary, cytotoxic chemotherapy may provide the extra time needed for those with good performance status to enroll into the next available clinical trial or to wait the approval of the next experimental agent.

In the adjuvant setting, chemotherapy remains experimental and should not be routinely used, given the lack of survival benefit demonstrated thus far, the added toxicity, and the concerns of lower response rates to immunotherapy 
in case of disease recurrence. It is reasonable to believe that the limited data available, heterogeneous patient population, and lack of prospective randomized clinical trials have precluded the identification of a subgroup of high-risk patients that truly benefit from chemotherapy. Unfortunately, it is unlikely that prospective randomized trials evaluating the role of chemotherapy (alone) in this rare patient population will be pursued, particularly now, when immune checkpoint inhibitors have proven safe and active in MCC patients. In fact, the next generation of trials will be looking into the addition of immune checkpoint inhibitors in the curative intent setting to optimize the outcome in these patients.

\section{Disclosure}

Research support to institution: EMD Serono, Exelixis, and Millenium. Scientific advisory board and consultant: EMD Serono and Pfizer. The author reports no conflict of interest in this work.

\section{References}

1. Boyle F, Pendlebury S, Bell D. Further insights into the natural history and management of primary cutaneous neuroendocrine (Merkel cell) carcinoma. Int J Radiat Oncol Biol Phys. 1995;31(2):315-323.

2. Lemos BD, Storer BE, Iyer JG, et al. Pathologic nodal evaluation improves prognostic accuracy in Merkel cell carcinoma: analysis of 5823 cases as the basis of the first consensus staging system. J Am Acad Dermatol. 2010;63(5):751-761.

3. Allen PJ, Bowne WB, Jaques DP, Brennan MF, Busam K, Coit DG. Merkel cell carcinoma: prognosis and treatment of patients from a single institution. J Clin Oncol. 2005;23(10):2300-2309.

4. Tai PT, Yu E, Winquist E, et al. Chemotherapy in neuroendocrine/Merkel cell carcinoma of the skin: case series and review of 204 cases. $J$ Clin Oncol. 2000;18(12):2493-2499.

5. Iyer JG, Blom A, Doumani R, et al. Response rates and durability of chemotherapy among 62 patients with metastatic Merkel cell carcinoma. Cancer Med. 2016;5(9):2294-2301.

6. Bhatia S, Storer BE, Iyer JG, et al. Adjuvant radiation therapy and chemotherapy in Merkel cell carcinoma: survival analyses of 6908 cases from the national cancer data base. $J$ Natl Cancer Inst. 2016;108(9). pii: djw042.

7. Poulsen M, Rischin D, Walpole E, et al; Trans-Tasman Radiation Oncology Group. High-risk Merkel cell carcinoma of the skin treated with synchronous carboplatin/etoposide and radiation: a Trans-Tasman Radiation Oncology Group Study - TROG 96:07. J Clin Oncol. 2003;21(23):4371-4376.

8. Foote M, Veness M, Zarate D, Poulsen M. Merkel cell carcinoma: the prognostic implications of an occult primary in stage IIIB (nodal) disease. J Am Acad Dermatol. 2012;67(3):395-399.

OncoTargets and Therapy

\section{Publish your work in this journal}

OncoTargets and Therapy is an international, peer-reviewed, open access journal focusing on the pathological basis of all cancers, potential targets for therapy and treatment protocols employed to improve the management of cancer patients. The journal also focuses on the impact of management programs and new therapeutic agents and protocols on
9. Deneve JL, Messina JL, Marzban SS, et al. Merkel cell carcinoma of unknown primary origin. Ann Surg Oncol. 2012;19(7):2360-2366.

10. Poulsen MG, Rischin D, Porter I, et al. Does chemotherapy improve survival in high-risk stage I and II Merkel cell carcinoma of the skin? Int J Radiat Oncol Biol Phys. 2006;64(1):114-119.

11. Poulsen M, Walpole E, Harvey J, et al. Weekly carboplatin reduces toxicity during synchronous chemoradiotherapy for Merkel cell carcinoma of skin. Int J Radiat Oncol Biol Phys. 2008;72(4):1070-1074.

12. Hasan S, Liu L, Triplet J, Li Z, Mansur D. The role of postoperative radiation and chemoradiation in merkel cell carcinoma: a systematic review of the literature. Front Oncol. 2013;3:276.

13. Mojica P, Smith D, Ellenhorn JD. Adjuvant radiation therapy is associated with improved survival in Merkel cell carcinoma of the skin. J Clin Oncol. 2007;25(9):1043-1047.

14. Lewis KG, Weinstock MA, Weaver AL, Otley CC. Adjuvant local irradiation for Merkel cell carcinoma. Arch Dermatol. 2006;142(6): 693-700.

15. Chen MM, Roman SA, Sosa JA, Judson BL. The role of adjuvant therapy in the management of head and neck merkel cell carcinoma: an analysis of 4815 patients. JAMA Otolaryngol Head Neck Surg. 2015; 141(2):137-141.

16. National Comprehensive Cancer Network. NCCN guidelines version 1.2017 merkel cell carcinoma. 2017. Available from: https://www. nccn.org/professionals/physician_gls/f_guidelines.asp\#mcc. Accessed May 17, 2017.

17. Paulson KG, Iyer JG, Blom A, et al. Systemic immune suppression predicts diminished Merkel cell carcinoma-specific survival independent of stage. J Invest Dermatol. 2013;133(3):642-646.

18. Heath M, Jaimes N, Lemos B, et al. Clinical characteristics of Merkel cell carcinoma at diagnosis in 195 patients: the AEIOU features. $J \mathrm{Am}$ Acad Dermatol. 2008;58(3):375-381.

19. Feng H, Shuda M, Chang Y, Moore PS. Clonal integration of a polyomavirus in human Merkel cell carcinoma. Science. 2008;319(5866): 1096-1100.

20. Starrett GJ, Marcelus C, Cantalupo PG, et al. Merkel cell polyomavirus exhibits dominant control of the tumor genome and transcriptome in virusassociated merkel cell carcinoma. MBio. 2017;8(1). pii:e02079-16.

21. Lipson EJ, Vincent JG, Loyo M, et al. PD-L1 expression in the Merkel cell carcinoma microenvironment: association with inflammation, Merkel cell polyomavirus and overall survival. Cancer Immunol Res. 2013;1(1):54-63.

22. Nghiem PT, Bhatia S, Lipson EJ, et al. PD-1 Blockade with pembrolizumab in advanced merkel-cell carcinoma. $N$ Engl $J$ Med. 2016;374(26):2542-2552.

23. Kaufman HL, Russell J, Hamid O, et al. Avelumab in patients with chemotherapy-refractory metastatic Merkel cell carcinoma: a multicentre, single-group, open-label, phase 2 trial. Lancet Oncol. 2016;17(10): 1374-1385.

24. Kaufman HL, Russell JS, Hamid O, et al. Durable responses to avelumab (anti-PD-L1) in patients with Merkel cell carcinoma progressed after chemotherapy: 1-year efficacy update. Presented at: AACR Annual Meeting; April 2-5, 2017; Washington, DC. Abstract CT079.

25. D'Angelo SP, Russell J, Hassel JC, et al. First-line (1L) avelumab treatment in patients (pts) with metastatic Merkel cell carcinoma (mMCC): preliminary data from an ongoing study. J Clin Oncol. 2017;35(15 Suppl):9530.

\section{Dovepress}

patient perspectives such as quality of life, adherence and satisfaction. The manuscript management system is completely online and includes a very quick and fair peer-review system, which is all easy to use. Visit http://www.dovepress.com/testimonials.php to read real quotes from published authors. 